\title{
Evidence-based Drug Dentistry Resource: New Initiative Project in Saudi Arabia
}

\author{
Yousef Ahmed Alomi* (D) BSC. \\ Pharm, MSc. Clin Pharm, BCPS, BCNSP, \\ DiBA, CDE \\ Critical Care Clinical Pharmacists TPN \\ Clinical Pharmacist, Freelancer Business \\ Planner, Content Editor, and Data Analyst, \\ Riyadh, SAUDI ARABIA. \\ Hanin Sumaydan Saleam Aljohani \\ Ministry of Health, Riyadh, SAUDI ARABIA. \\ Arub Abdullah Batil Albatil \\ Majmaah University, College of Dentistry \\ Faculty of Dentistry at Al Zulfi Riyadh, \\ SAUDI ARABIA.
}

\section{Correspondence:}

Dr. Yousef Ahmed Alomi, BSc. Pharm, MSc. Clin Pharm, BCPS, BCNSP, DiBA, CDE Critical Care Clinical Pharmacists TPN Clinical Pharmacist, Freelancer Business Planner, Content Editor, and Data Analyst, P.O.BOX 100, Riyadh 11392, Riyadh, SAUDI ARABIA.

Phone no: +966504417712 E-mail:yalomi@gmail.com

Received: 20-12-2020

Approved: 29-2-2021

Copyright: ๑ the author(s),publisher and licensee Pharmacology, Toxicology and Biomedical Reports. This is an open-access article distributed under the terms of the Creative Commons Attribution NonCommercial License, which permits unrestricted non-commercial use, distribution, and reproduction in any medium, provided the original work is properly cited.

This is an open access article distributed under the terms of the Creative Commons Attribution-NonCommercial-ShareAlike 4.0 License

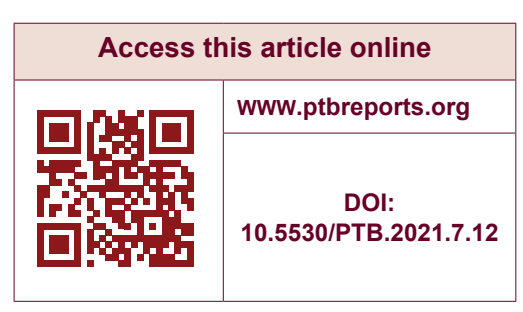

\begin{abstract}
Objectives: Evidence-based dentistry is a existing practice in the dental care. Evidence-based dental medications play a grave part in this field. The reconnoiter of evidence-based dental drug information resources is swotted in this topic. Methods: It is a chronicle review of evidence-based dentistry of drug information resources. The literature had been examined through PubMed and Medline from the 1960s to 2021s. All types of literature encompassed. If not existed, the textbook of drug information will be designed. The project is defined through market analysis and SWOT tools. The team and cost management will be deliberated. Quality management and risk factors will be involved in the project. The list of evidence-based dentistry discovers literature coverage, frequency of updating, type of publications online or applications, and responsible publisher. Results: There are more than twenty evidence-based dentistry drug information resources were nominated. There are numerous types of resources, including free-of-charge evidence-based. It contained of collecting the primary literature and evidence-based dental guidelines. Some resources had a collection of meta-analysis and systemic review literature with a paid subscription. Other comprehensive collection of journal publications includes from the USA and European journals. Additional drug information resources through a comprehensive search engine of all evidence-based dental drug information resources. The choice of evidence-based dentistry is based on numerous factors debated in the topic. Conclusion: Evidence-based dentistry with an emphasis on drug information resources connected issues is a present part of dental practice. Therefore, the implementation of the project is mandatory at the healthcare organizations in the dental care.

Key words: Evidence-based, Drug, Dentistry, Resources, Saudi Arabia.
\end{abstract}

\section{INTRODUCTION}

Evidence-based medicine is perilous and measured an essential tool employed in the healthcare practice. Each healthcare provider had an evidence-based field, counting evidence-based dentistry, evidence-based nursing, and evidencebased pharmacy. The evidence-based tools necessity specific drug information references or resources. Those references are applied to implement evidence-based knowledge and practice in the healthcare field. Those references came as textbooks, online or website, or mobile applications. All evidence-based drug information resources are a portion of evidence-based medicine. The dentists among healthcare professionals exploited evidence-based medications in dental care. Failing to provide precise drug information may have numerous negative consequences. It is projected that one-third to half of all drugs employed worldwide are thrown away, posing a financial and health danger. ${ }^{1}$ Also, the center offers an in-depth, unbiased source of critical drug knowledge; to meet the needs of practicing physicians, pharmacists, and other health care practitioners. Moreover, financial and legal interests make the pharmacist's position more protuberant in society and culture to defend the patient's health. ${ }^{2}$ Unfortunately, the knowledge of evidence-based drug information resources was intolerable. ${ }^{3}$ As a result, a literature review of evidence-based dentistry accenting drug information resources is highly suggested. Many studies conversed EBD, and authors are not acquainted with any investigation about evidence-based drug information references locally or the Middle East. ${ }^{4}$ The goal of the current review is to reconnoiter the evidencebased dental drug information resources.

\section{METHODS}

It is a literature review of drug information references website for the dentist. Instead, drug information resource websites are used during the dental care. The PubMed search includes drug information resources website and the dentist or drug information resources website and dentistry or drug information references website. The word is employed as a general term, not a subject term. The search comprised all types of studies Clinical Study, Clinical Trial, Clinical Trial, Phase I, Clinical Trial, Phase II, Clinical Trial, Phase III, Clinical Trial, Phase IV, Controlled Clinical Trial, Meta-Analysis, Observational Study, Practice Guideline, Randomized Controlled Trial, Review, and Systematic Review. The searching date from 1985 to May 2021. The PubMed search words counting as follows:

\section{Evidence-based Dental Drug} Information Filters: Full Text, Humans, English

(("evidence"[All Fields] OR “evidences"[All Fields] OR "evident"[All Fields] OR "evidently"[All Fields]) AND ("based"[All 
Fields] OR "basing”[All Fields]) AND ("dental health services"[MeSH Terms] OR ("dental"[All Fields] AND "health"[All Fields] AND "services"[All Fields]) OR "dental health services"[All Fields] OR "dental"[All Fields] OR "dentally"[All Fields] OR "dentals"[All Fields]) AND “drug”[All Fields] AND ("inform”[All Fields] OR "informal"[All Fields] OR "informality"[All Fields] OR "informally"[All Fields] OR "informant"[All Fields] OR "informant s"[All Fields] OR “informants"[All Fields] OR "information”[All Fields] OR “information $s$ "[All Fields] OR “informational”[All Fields] OR “information"[All Fields] OR “informative"[All Fields] OR “informatively"[All Fields] OR “informativeness" [All Fields] OR "informativity”[All Fields] OR “informed"[All Fields] OR “informer"[All Fields] OR "informers"[All Fields] OR “informing”[All Fields] OR “informs”[All Fields])) AND ((fft[Filter]) AND (humans[Filter]) AND (english[Filter]))

\section{Evidence-based Dentist Drug Information Filters: Full Text, Humans, English}

((“evidence"[All Fields] OR “evidences"[All Fields] OR “evident"[All Fields] OR "evidently"[All Fields]) AND ("based"[All Fields] OR "basing"[All Fields]) AND ("dentist s"[All Fields] OR "dentists"[MeSH Terms] OR “dentists"[All Fields] OR “dentist”[All Fields]) AND "drug"[All Fields] AND ("inform"[All Fields] OR "informal"[All Fields] OR “informality”[All Fields] OR “informally”[All Fields] OR “informant"[All Fields] OR "informant s"[All Fields] OR "informants"[All Fields] OR “information"[All Fields] OR “information"[All Fields] OR “informational”[All Fields] OR “informations"[All Fields] OR “informative"[All Fields] OR "informatively"[All Fields] OR "informativeness"[All Fields] OR “informativity"[All Fields] OR “informed”[All Fields] OR “informer"[All Fields] OR “informers"[All Fields] OR “informing”[All Fields] OR “informs"[All Fields])) AND ((fft[Filter]) AND (humans[Filter]) AND (english[Filter]))

\section{Evidence-based Dentistry Drug Information Filters: Full Text, Humans, English}

((“evidence based dentistry"[MeSH Terms] OR ("evidence based"[All Fields] AND "dentistry"[All Fields]) OR "evidence based dentistry"[All Fields] OR ("evidence"[All Fields] AND "based"[All Fields] AND "dentistry"[All Fields]) OR "evidence based dentistry"[All Fields]) AND "drug"[All Fields] AND ("inform”[All Fields] OR "informal”[All Fields] OR "informality"[All Fields] OR “informally"[All Fields] OR “informant”[All Fields] OR "informant s"[All Fields] OR “informants"[All Fields] OR “information”[All Fields] OR “information”[All Fields] OR “informational”[All Fields] OR “informations"[All Fields] OR "informative"[All Fields] OR “informatively"[All Fields] OR "informativeness"[All Fields] OR "informativity"[All Fields] OR "informed"[All Fields] OR "informer"[All Fields] OR “informers"[All Fields] OR “informing”[All Fields] OR “informs"[All Fields])) AND ((fft[Filter]) AND (humans[Filter]) AND (english[Filter]))

As a result, no studies came up with it. Another search had been done if pharmacy textbooks of drug information and some google searching engine. The authors employed drug information resources as websites or drug information resources websites for dental medication inquiries and secondary or tertiary drug information resources. The author intended some parameters to recapitulate the drug information references suitable for the dental care. Each resource summary entailed of name of resource, content, inquiries answered, type of resources, number of medications or journal covered, frequently update, last edition, price, subscription. This also includes publisher, manual book, online version, website address, application type, usage of dental student recommendations, usage of the general dental practitioner, usage of specialized or consultant dentist and dentistry specialties.

\section{RESULTS}

\section{Assessment of Needs}

Drug information resources deliver clinicians with safer medications and play a vivacious role in improving drug safety. The drug information resources should be well equipped with all the essential resources for providing comprehensive, recent, and up-to-date information on medication queries. ${ }^{5}$ Corresponding to the augmented acceptance of drug use in dental treatment, dentists need to be warned to potential problems resulting from the drug therapy. The practice of dentistry today has advanced in pharmacology and toxicology. It is no fortune that dental school curricula revisions include noteworthy teaching time dedicated to studying drugs and their application. ${ }^{6}$ The effectual use of a drug information website is indispensable for all healthcare providers regardless of their practice site. The provision of balanced information can help in weakening the occurrence of drug-related complications and pledge drug safety to an extent. ${ }^{7-9}$

These people come to a wide collection of medical conditions and drug treatments, many of which interact with the therapeutic dental milieu. The dentist staff should supply the wanted drug-use information in these areas. One of the conservative methods of new project assessment is a SWOT analysis. It means strengths, weaknesses, opportunities, and threats of the project. ${ }^{10}$ Evaluating each resource as accessible earlier may be time-consuming. Often, the responses are desired quickly, which may force one to rely only on the top results, leading to decisions based on a snapshot of evidence. The weaknesses of this project are overlooking this service, no education or guidance in the usage of the drug information center, difficulties in supporting and funding these services. The threats to this project includes no dentist concerned in this field and the plan.

\section{Project Description}

The scope of the existing topic is to reconnoiter evidence-based dentistry highlighting the drug information resources. The project's vision is to grasp the best with high-quality, evidence-based dentistry drug information at realistic prices. The mission of the project is to deliver cost-effective drug information resources for evidence-based dentistry. In addition, the project intentions to declare the updated evidence-based dentistry drug information's references, offer education and training for a dental practitioner, and evaluate the clinical and economic impact of evidence-based dentistry drug information's resources. The project wants to be organized by the management team; the experts in the team should accomplish the dentist drug information center: the team contains of clinical pharmacists, expert pharmacists, and pharmacy technician is skilled in drug information, dentists, dentists assistant, and nursing. The team should educate and train the dentist and dentist's assistant in drug information-related issues. The team should set up new policies and procedures connected to the drug information services, monitor the program's implementation, and measure the outcome with the economic influence of the new services. One of the essential things is to implement the drug information services (financial budget). The budget should reflect cost-related issues, counting education and training. Besides, the cost of equipment is desirable for the preparation and the management team meetings. The defined budget should be observed throughout the project period implementation. The dentist staff wants to appear several courses on searching drug information resources and how to pick the fitting references for any dental drug information inquiries. Besides that, the education on drug information centers is stability research. Education and training are vital for dentist staff. Additional educational course needs for administrative officers. It contains managing team and higher administration and coverage of health insurance. The projected demand to effort on dental quality management tools called Balance Scored Card as key performance indicators about dental drug 


\begin{tabular}{|c|c|c|c|c|c|c|c|c|c|}
\hline 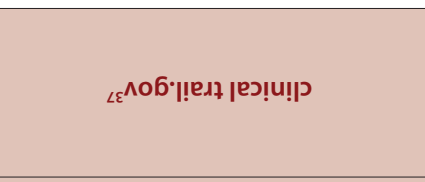 & 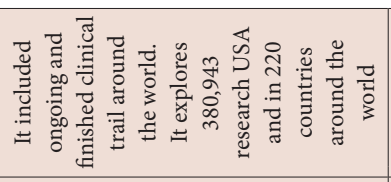 & 六 & , & 离 & 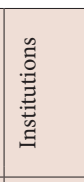 & $\check{z}$ & $\cong$ & $\check{z}$ & 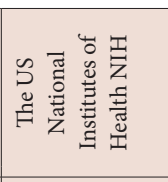 \\
\hline 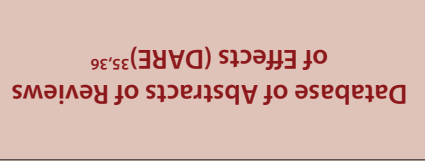 & 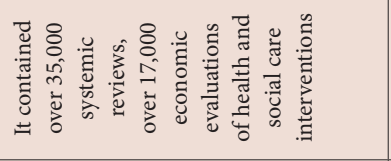 & 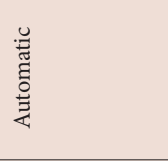 & & 营 & 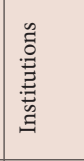 & 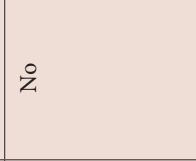 & $\approx$ & $\stackrel{\circ}{z}$ & 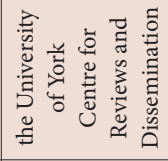 \\
\hline 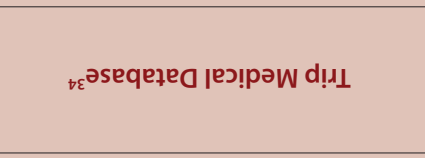 & 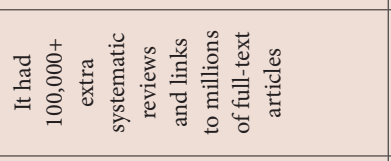 & 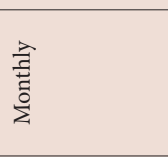 & & 胥 & 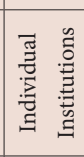 & $\stackrel{\circ}{2}$ & 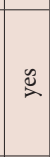 & $\stackrel{\gtrless}{2}$ & 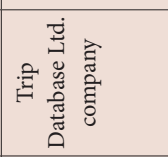 \\
\hline 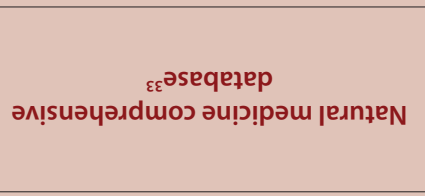 & 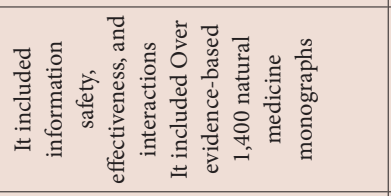 & 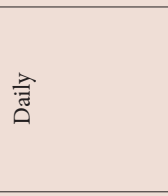 & & 苛 & 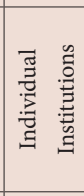 & そ̊ & $\approx$ & $\stackrel{\circ}{2}$ & 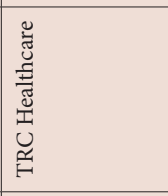 \\
\hline 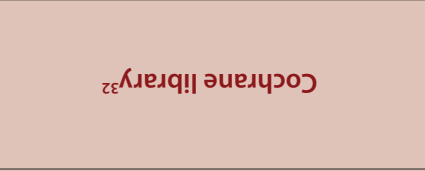 & 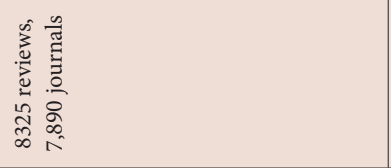 & 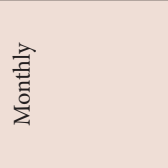 & & 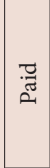 & 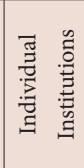 & 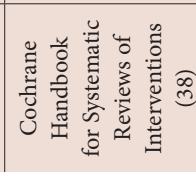 & $\dddot{\varkappa}$ & $\cong$ & $\frac{2}{3}$ \\
\hline 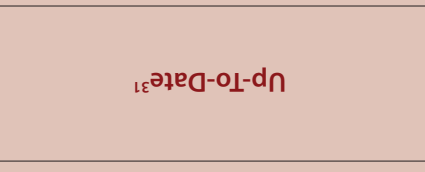 & 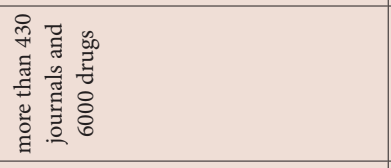 & 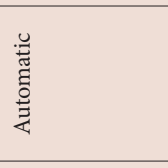 & $\Xi$ & 节 & 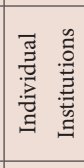 & $\grave{z}$ & $\cong$ & $\cong$ & 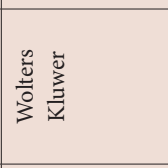 \\
\hline 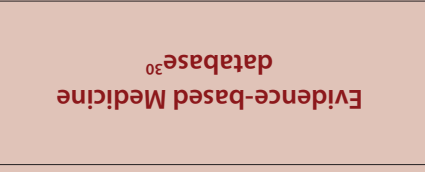 & 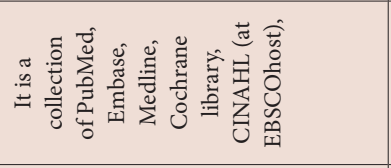 & 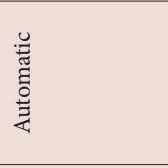 & 吾 & 苞 & 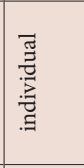 & $\stackrel{8}{2}$ & $\cong$ & $\grave{z}$ & $\begin{array}{l}0 \\
0 \\
0 \\
\text { M. }\end{array}$ \\
\hline бгрәшеика & 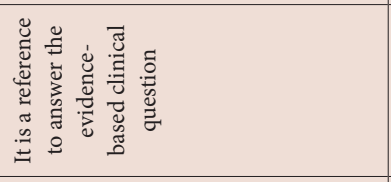 & 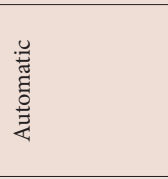 & 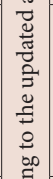 & : & 总 & $\AA$ & $\cong$ & 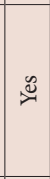 & 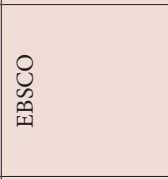 \\
\hline 8z & 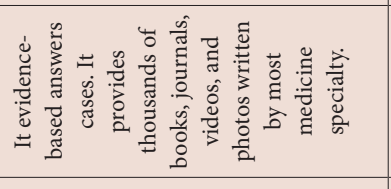 & 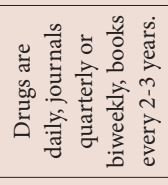 & 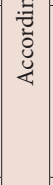 & 胥 & 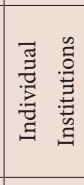 & $\stackrel{\circ}{z}$ & 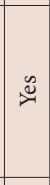 & 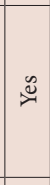 & 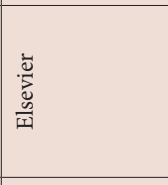 \\
\hline 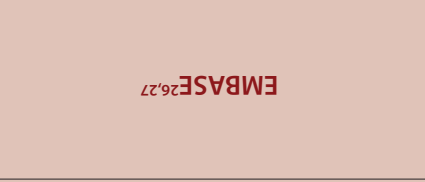 & 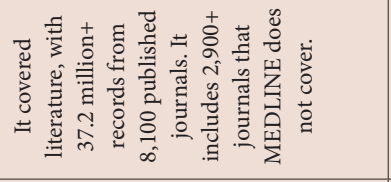 & $\frac{5}{3}$ & & 苛 & 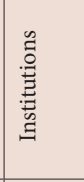 & $\stackrel{\circ}{z}$ & $\cong$ & $\grave{z}$ & 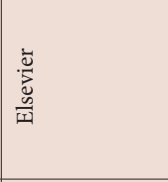 \\
\hline 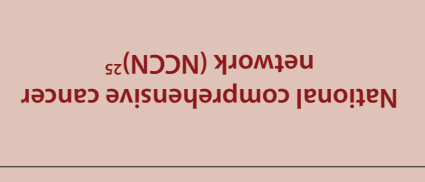 & 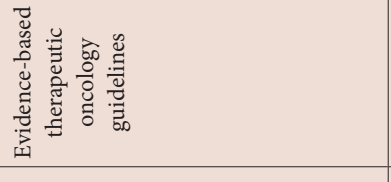 & 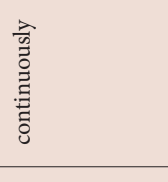 & & 号 & 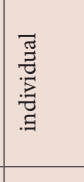 & $\stackrel{\circ}{z}$ & 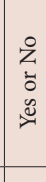 & $\approx$ & 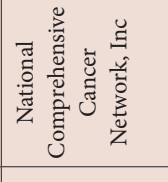 \\
\hline 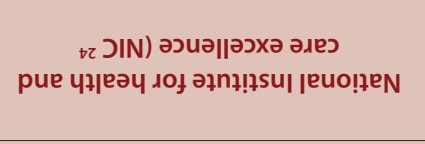 & 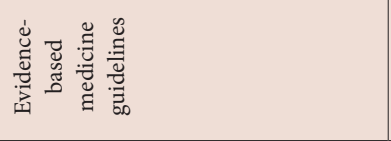 & 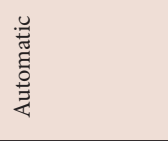 & & 㞻 & 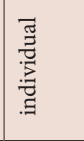 & 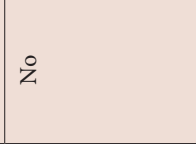 & $\cong$ & ๕ & 恾 \\
\hline 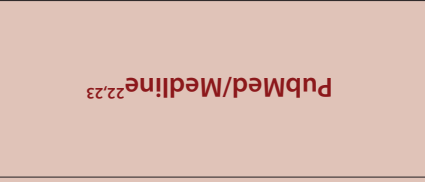 & 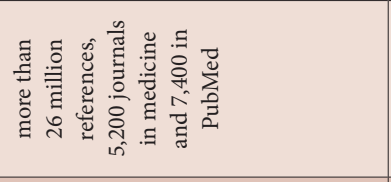 & $\frac{2}{3}$ & & ฆั & 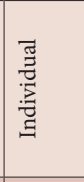 & $\stackrel{8}{z}$ & $\stackrel{2}{2}$ & $\cong$ & 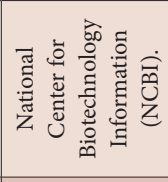 \\
\hline & 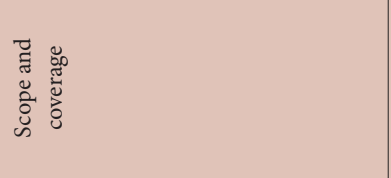 & 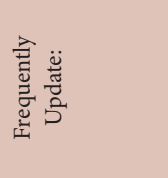 & 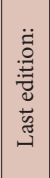 & $\ddot{\ddot{g}}$ & 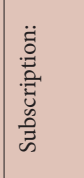 & 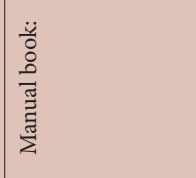 & 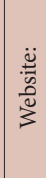 & 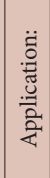 & 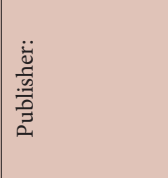 \\
\hline
\end{tabular}




\begin{tabular}{|c|c|c|c|c|c|c|c|c|c|c|}
\hline & ${ }_{s s} \triangle W \times O$ Кq реәу & 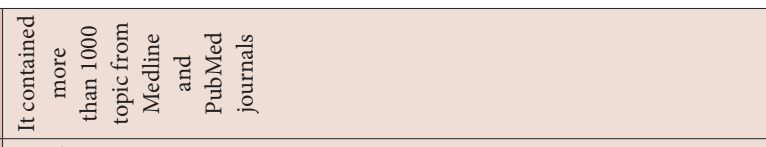 & 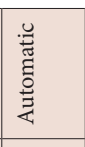 & & 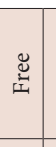 & 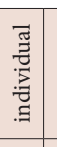 & $\gtreqless$ & 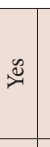 & $\cong$ & 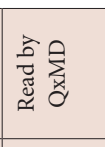 \\
\hline & 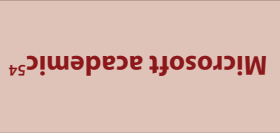 & 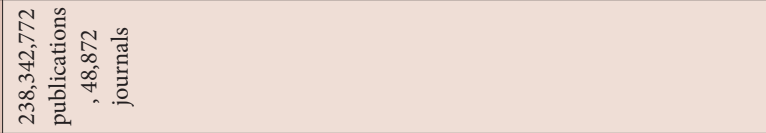 & 䶀 & & 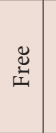 & 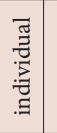 & そ & $\stackrel{\mathscr{C}}{2}$ & $\approx$ & 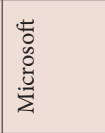 \\
\hline & 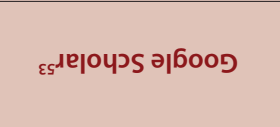 & 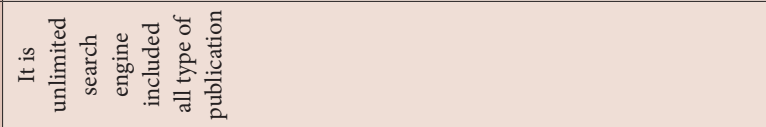 & 咅 & & 选 & 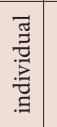 & $\stackrel{2}{2}$ & $\cong$ & $\cong$ & $\begin{array}{l}\frac{0}{\square 0} \\
\vdots \\
\dot{G}\end{array}$ \\
\hline & zs rWg & 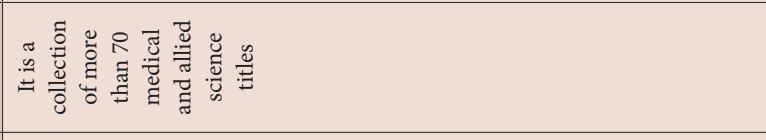 & 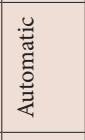 & & 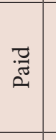 & 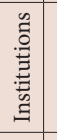 & 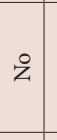 & 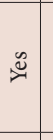 & $\stackrel{8}{z}$ & 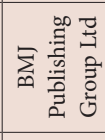 \\
\hline & เs sndors & 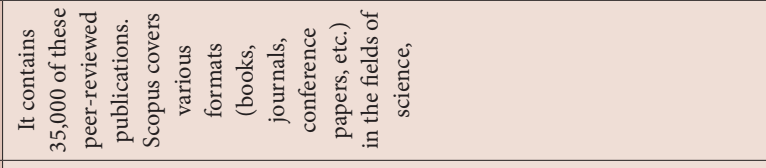 & 咅 & & $\stackrel{\vec{\pi}}{2}$ & 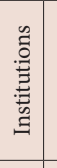 & $\stackrel{8}{z}$ & 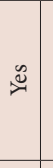 & $\stackrel{\circ}{z}$ & 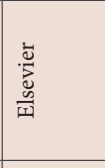 \\
\hline & 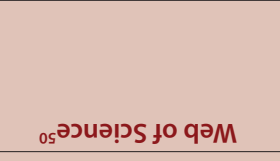 & 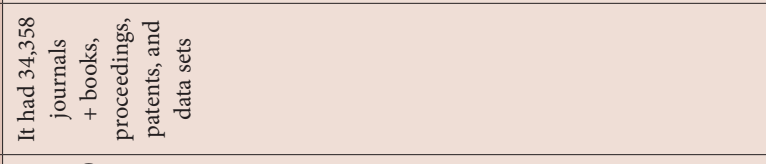 & 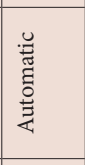 & & $\frac{\pi}{\pi}$ & 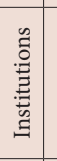 & そ & $\approx$ & $\gtreqless$ & 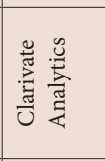 \\
\hline & 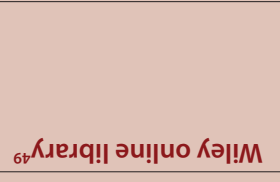 & 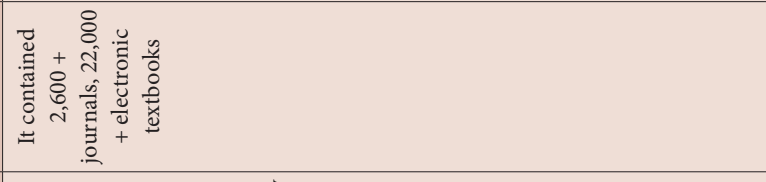 & 产 & $\mid$ & 范 & 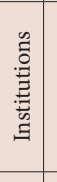 & 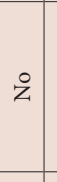 & $\cong$ & z & $\frac{\bar{a}}{3}$ \\
\hline & ${ }_{87 t}+5 ə n \delta 0 \lambda_{d}$ & 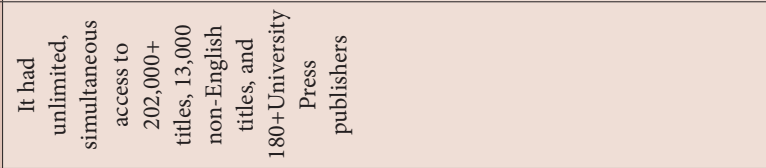 & 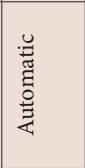 & $\mid$ & 胥 & 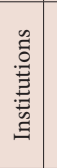 & 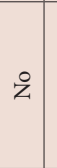 & 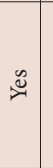 & $\stackrel{\circ}{z}$ & 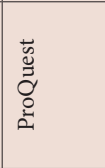 \\
\hline & ¿әәеqедер әбеs & 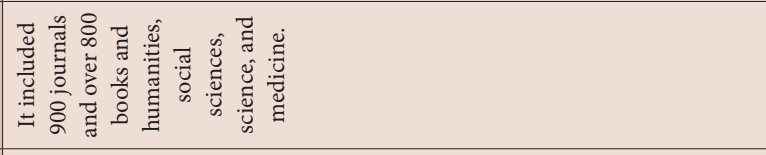 & 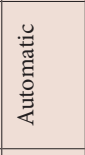 & 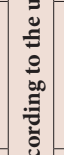 & 尝 & 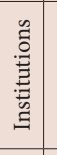 & ¿ & $\mathscr{\sim}$ & $\grave{z}$ & 荡 \\
\hline & ${ }_{9} ә$ әsеqедер дәби!ıds & 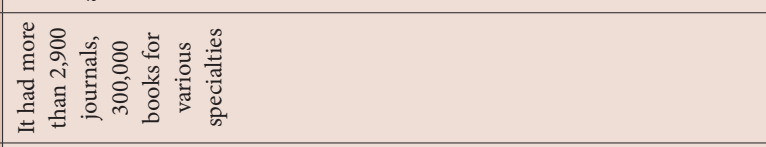 & 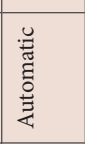 & \& & 缡 & 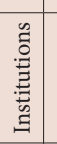 & そ & $\cong$ & 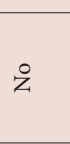 & 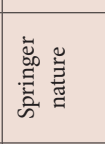 \\
\hline 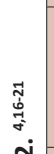 & 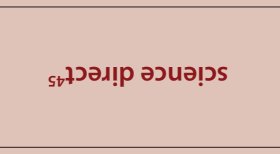 & 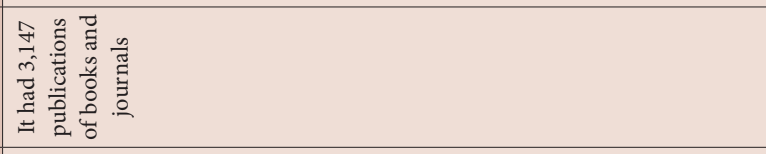 & 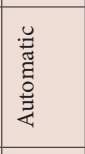 & & 可 & 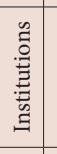 & $\stackrel{2}{2}$ & 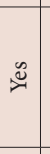 & $\grave{z}$ & 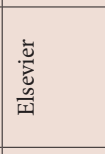 \\
\hline 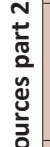 & 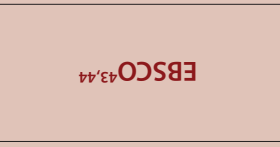 & 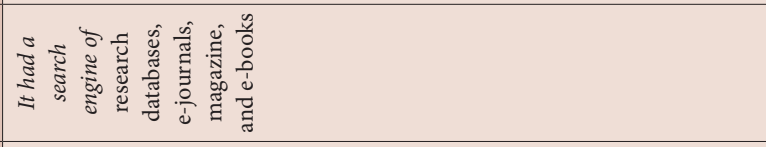 & 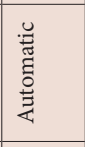 & & : & 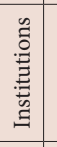 & 2 & 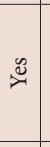 & $\cong$ & 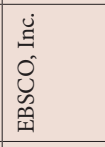 \\
\hline 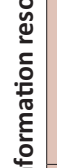 & 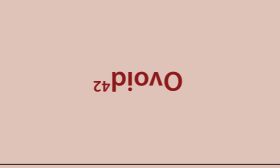 & 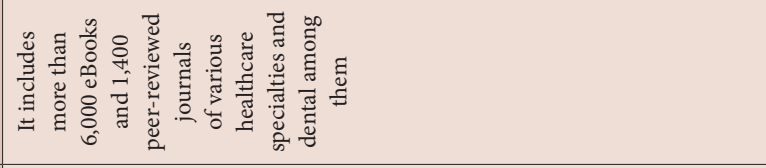 & 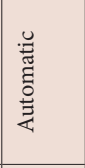 & & 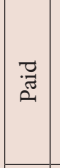 & 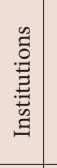 & $\stackrel{2}{2}$ & $\cong$ & $\grave{z}$ & 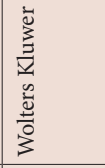 \\
\hline 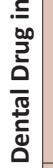 & כד כוH & 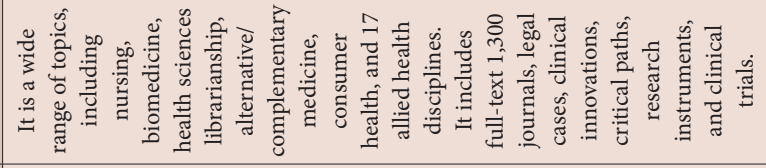 & 竞 & & 胥 & 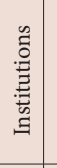 & ¿ & $\cong$ & $\grave{z}$ & 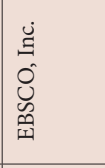 \\
\hline 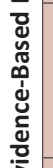 & 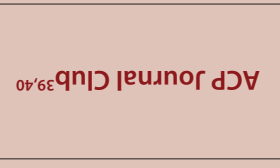 & 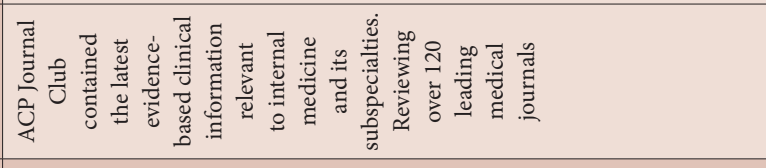 & 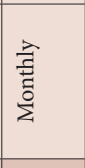 & & 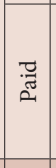 & 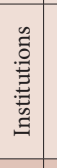 & ¿̊ & 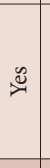 & 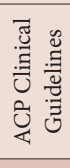 & \\
\hline 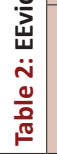 & & 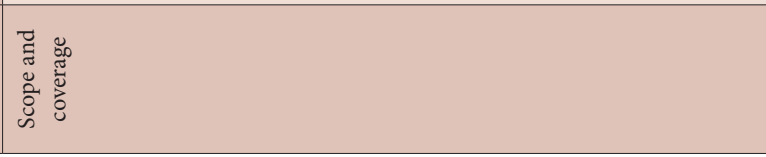 & 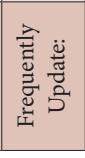 & 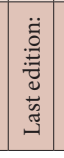 & $\ddot{\ddot{g}}$ & 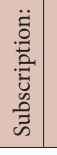 & 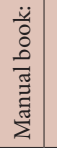 & 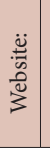 & 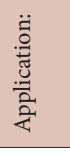 & 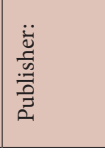 \\
\hline
\end{tabular}




\begin{tabular}{|c|c|c|c|c|c|c|c|c|c|}
\hline 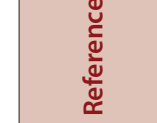 & $\begin{array}{l}\tilde{N} \\
\hat{\sim} \\
\hat{\sim}\end{array}$ & $\stackrel{H}{N}$ & $\stackrel{\text { L }}{\text { L }}$ & $\begin{array}{l}\hat{i} \\
\text { d̂. }\end{array}$ & $\stackrel{\infty}{\sim}$ & సิ & \multicolumn{2}{|l|}{$\stackrel{m}{ }$} & $\vec{m}$ \\
\hline 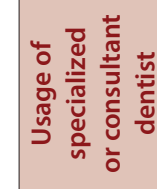 & 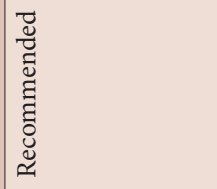 & 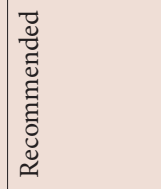 & 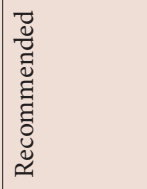 & 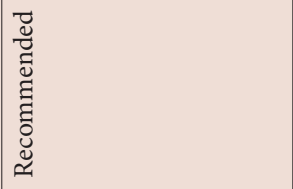 & 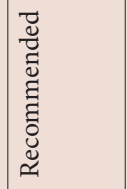 & 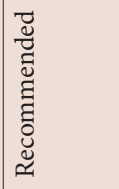 & \multicolumn{2}{|l|}{ 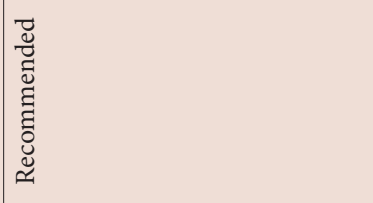 } & 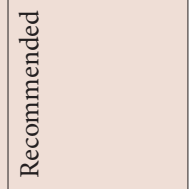 \\
\hline 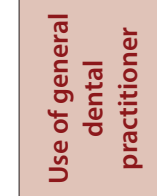 & 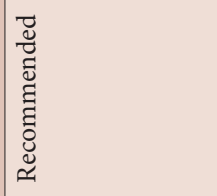 & 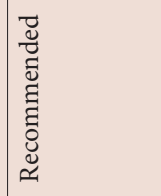 & 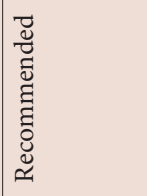 & 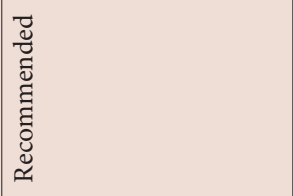 & 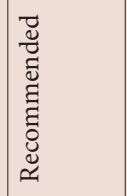 & 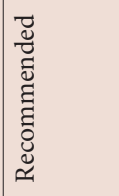 & \multicolumn{2}{|l|}{ 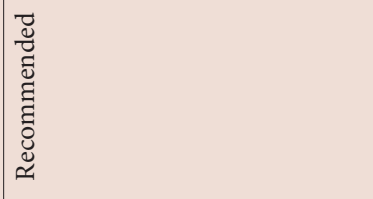 } & 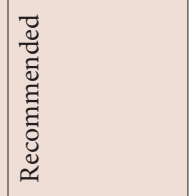 \\
\hline 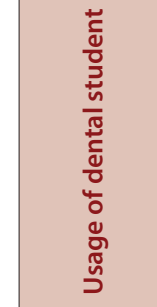 & 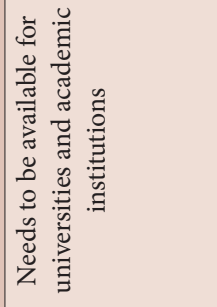 & 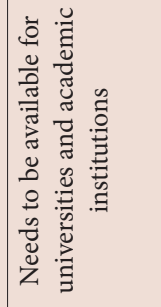 & 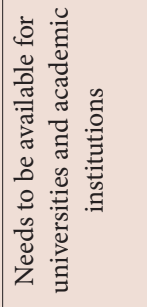 & 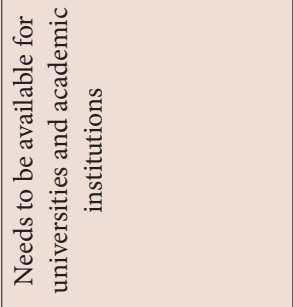 & 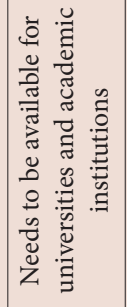 & 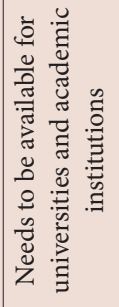 & \multicolumn{2}{|l|}{ 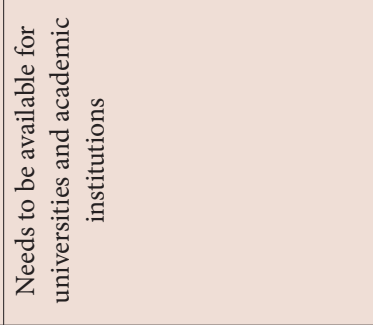 } & 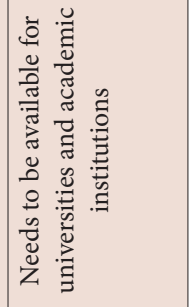 \\
\hline 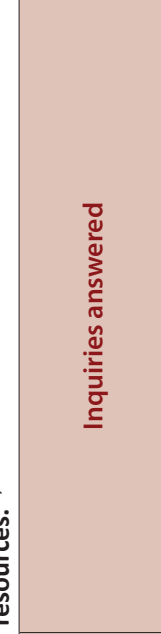 & 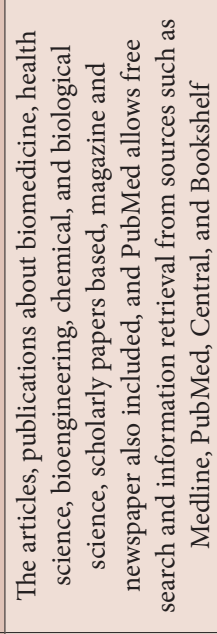 & 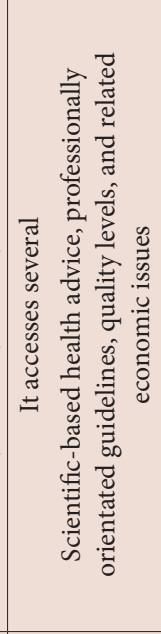 & 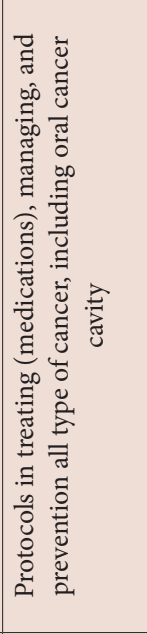 & 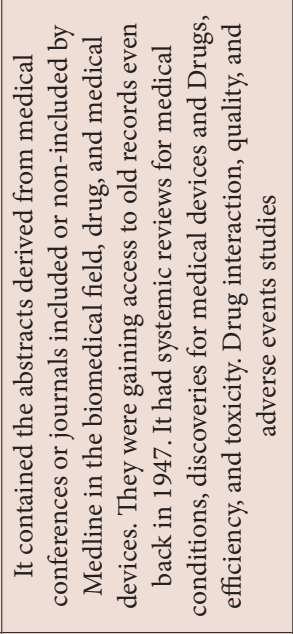 & 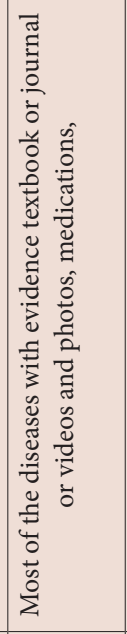 & 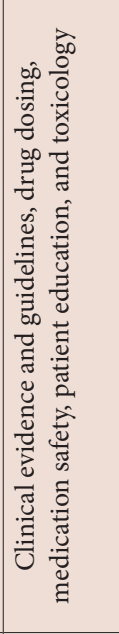 & \multicolumn{2}{|c|}{ 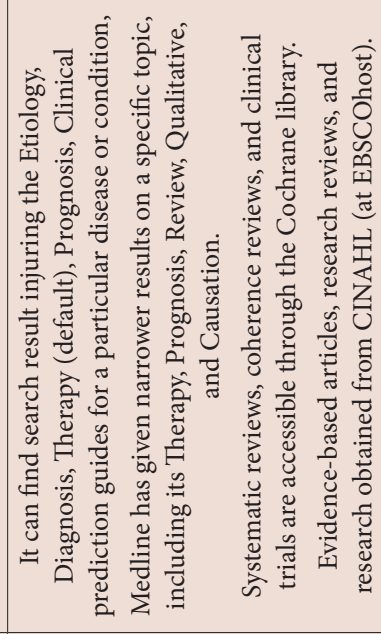 } & 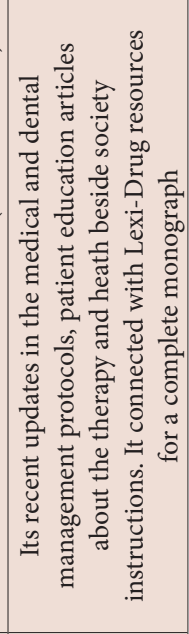 \\
\hline 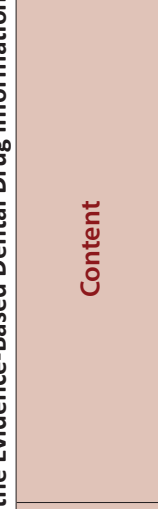 & 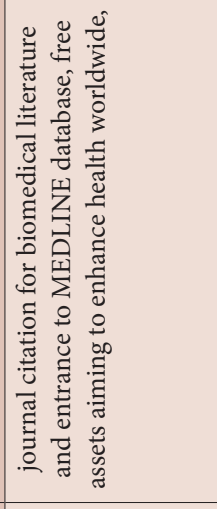 & 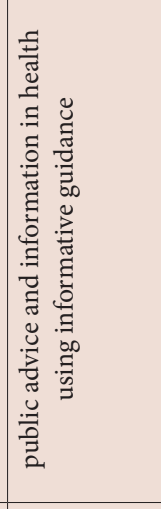 & 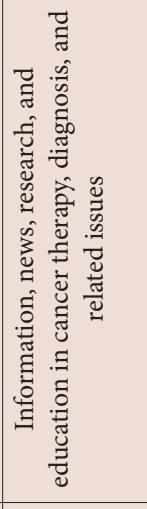 & 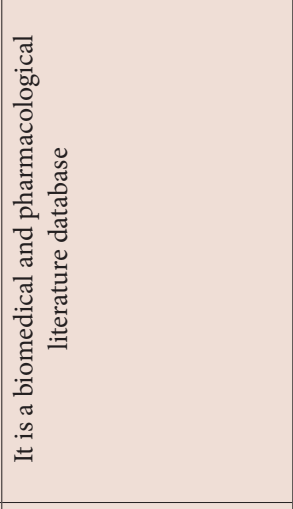 & 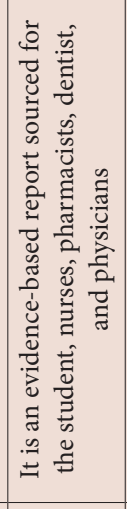 & 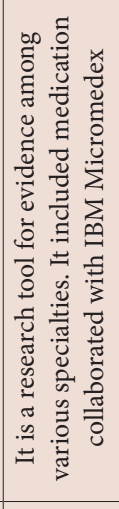 & \multicolumn{2}{|l|}{ 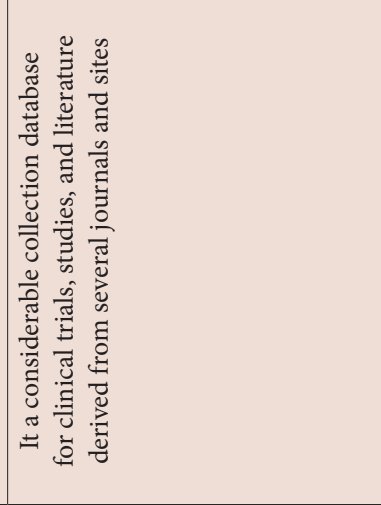 } & 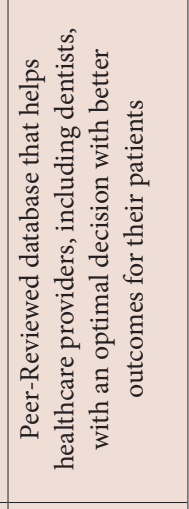 \\
\hline 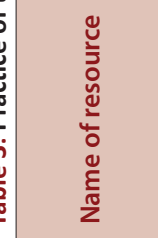 & 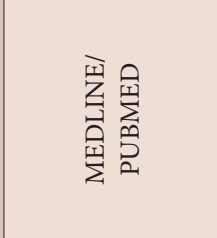 & 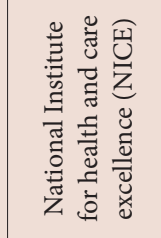 & 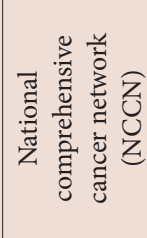 & $\begin{array}{l}\sum_{4}^{W} \\
\sum_{=4}^{\infty}\end{array}$ & 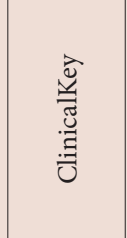 & 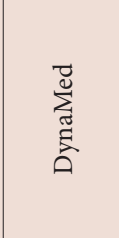 & \multicolumn{2}{|l|}{ 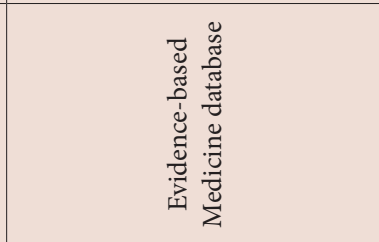 } & 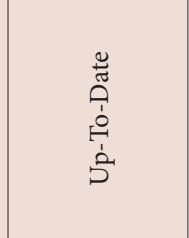 \\
\hline
\end{tabular}




\begin{tabular}{|c|c|c|c|c|c|c|c|c|c|c|}
\hline 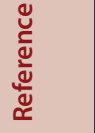 & $\tilde{m}$ & $m$ & mे & $\begin{array}{l}\infty \\
\tilde{m}^{2} \\
n^{2}\end{array}$ & m & 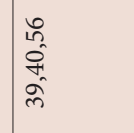 & $F$ & F & \begin{tabular}{l}
$\mathbb{Z}$ \\
\multirow{2}{*}{}
\end{tabular} & if \\
\hline 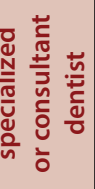 & 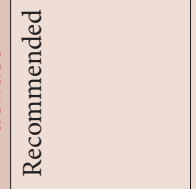 & 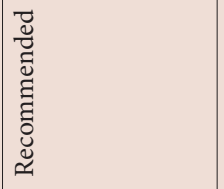 & 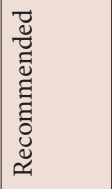 & 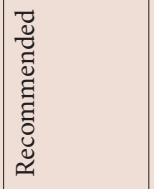 & 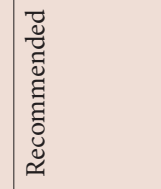 & 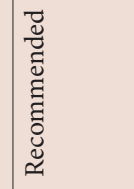 & 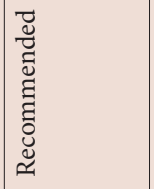 & 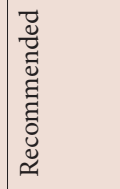 & 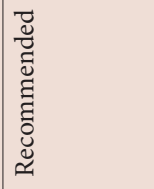 & 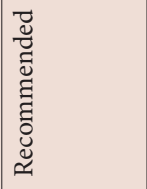 \\
\hline 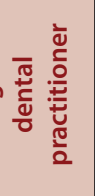 & 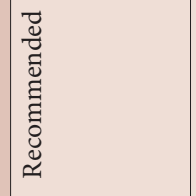 & 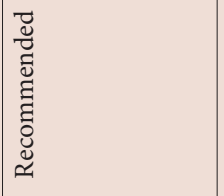 & 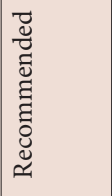 & 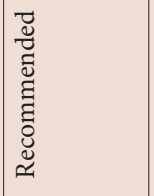 & 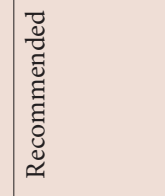 & 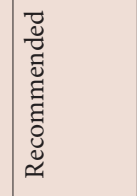 & 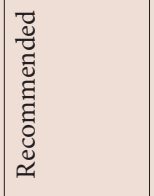 & 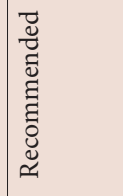 & 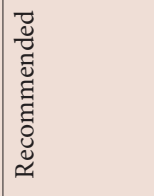 & 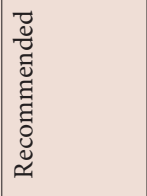 \\
\hline 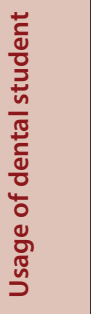 & 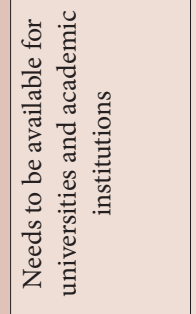 & 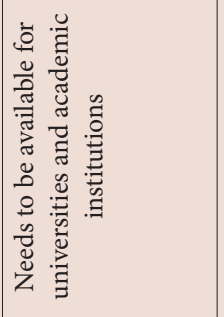 & 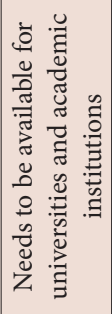 & 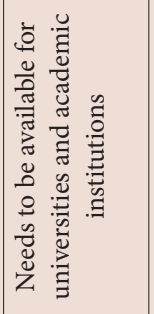 & 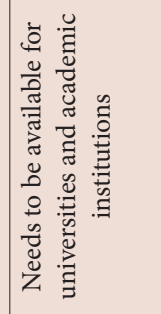 & 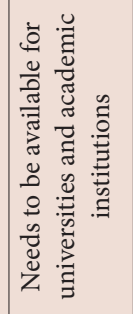 & 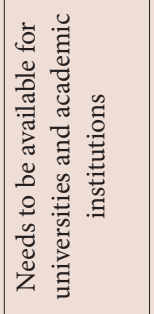 & 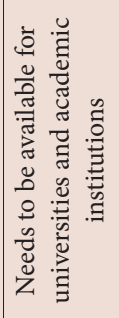 & 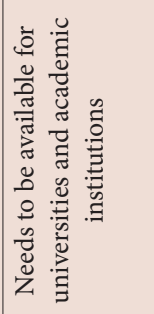 & 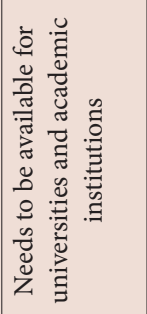 \\
\hline 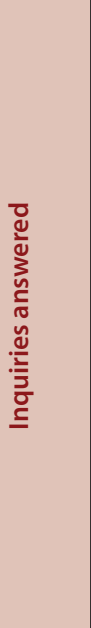 & 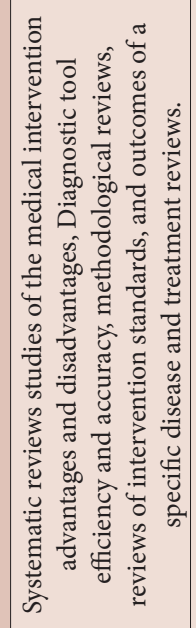 & 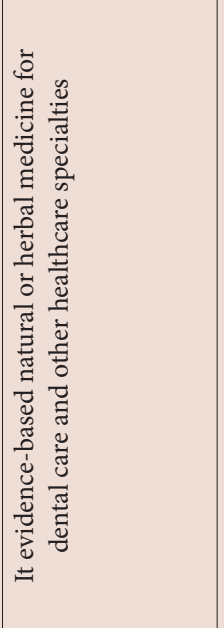 & 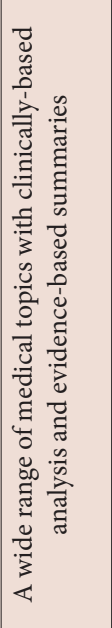 & 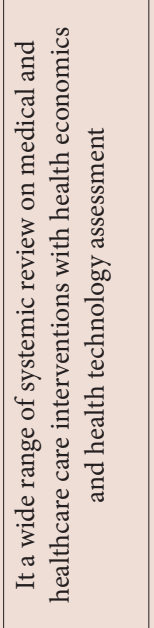 & 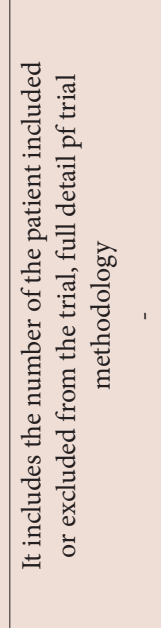 & 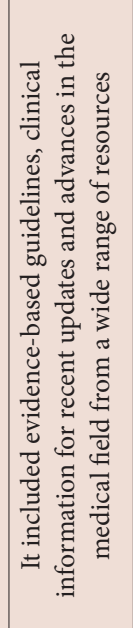 & 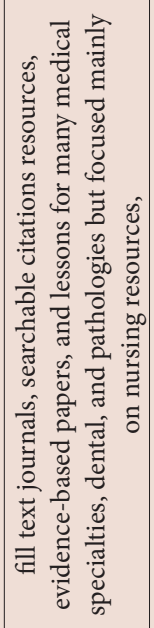 & 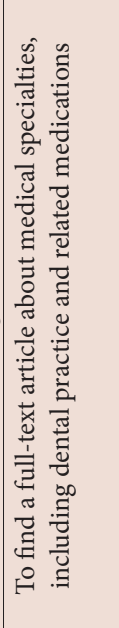 & 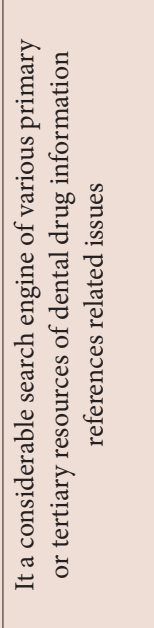 & 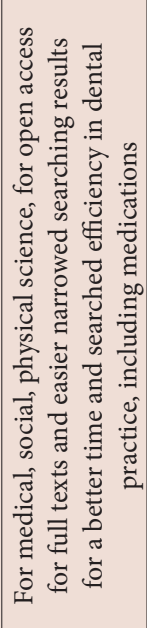 \\
\hline 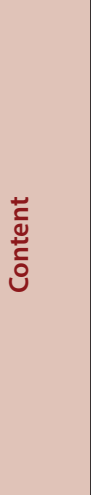 & 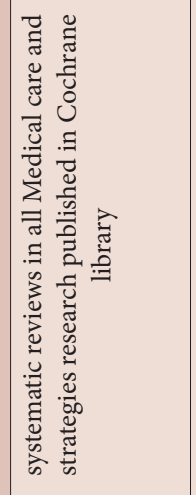 & 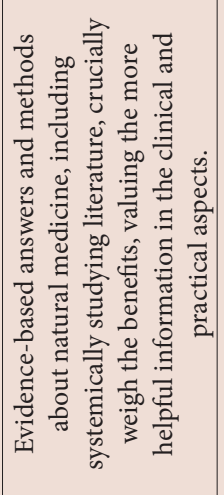 & 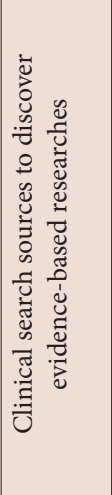 & 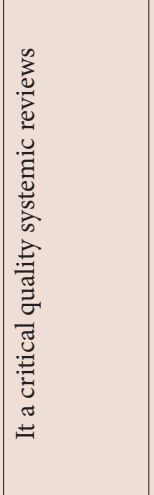 & 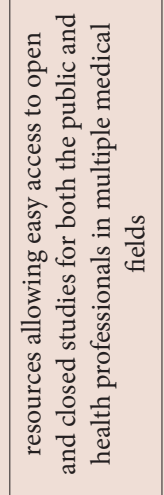 & 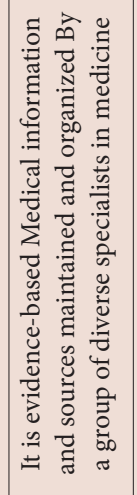 & 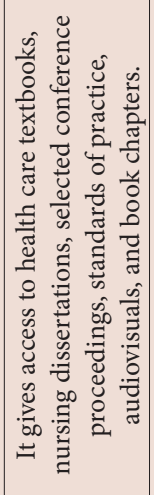 & 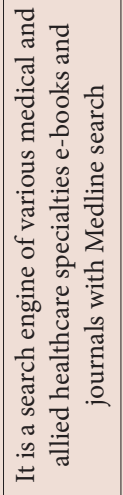 & 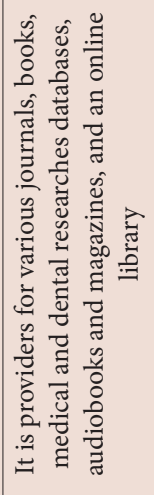 & 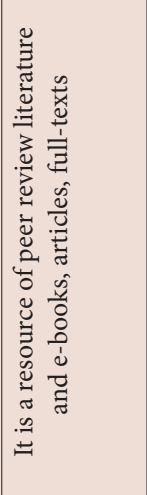 \\
\hline 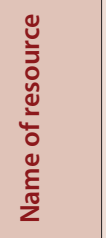 & 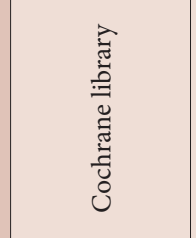 & 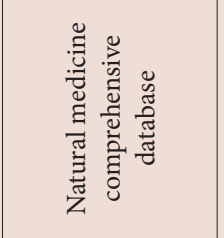 & 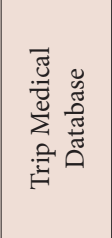 & 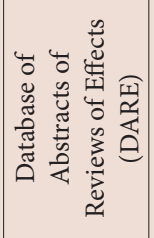 & 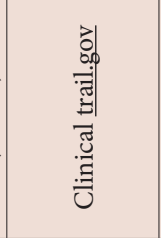 & 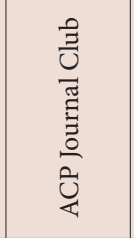 & 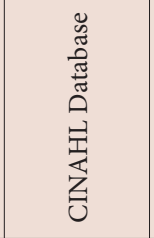 & $\overrightarrow{\bar{\sigma}}$ & $\begin{array}{l}\text { О } \\
\text { Oొ } \\
\text { I. }\end{array}$ & 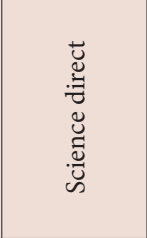 \\
\hline
\end{tabular}




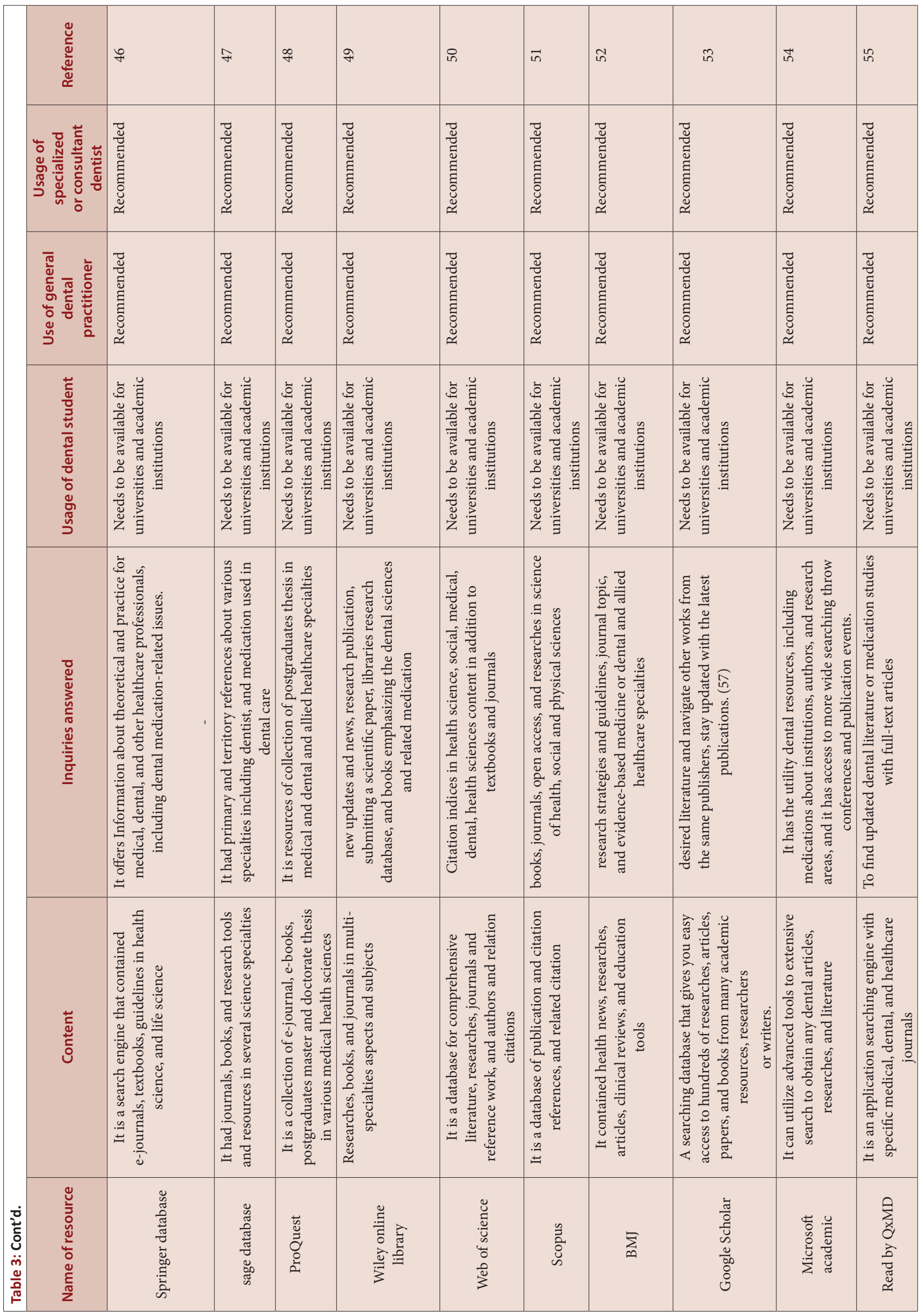


information resources websites with multiple directions, counting the customer as dentists, finance, internal process, education, and innovation. ${ }^{11}$ Risks includes in the current project: budget, scope, personnel, scheduled, technical, and quality risks. This project might be uncovered to personnel risk due to the shortage of expert dentists. It might also be exposed to budget risk. The demand of a budget includes education and training of the dentist staff with multiple experiences. This project might also be exposed to technical risks such as material is not being accessible. Quality risks are met due to the untrained dentist being available. ${ }^{12,13}$ More than twenty-eight evidence-based dentistry drug information resources were nominated (Table 1, 2 and 3).

\section{DISCUSSION}

Over twenty years ago of beginning evidence-based medicine. Evidencebased specialties like evidence-based dysentery followed them. ${ }^{14}$ All dental practitioners need to implement the concept. However, the dentist requests some resources with importance on drug information resources. As a result, the existing review of dental evidence-based drug information references is defensible. There are many EBD for the drug information. Some of the references are free of variations, and any dental student or any level of dental practitioner can use them like PubMed/ Medline (which is more frequently used by the dentist), ${ }^{15}$ Nice, and NCCN. Other paid resources look like them with general review and deliberated the medical or dental topics based on evidence with recorded recommendations; it is called update (which is less often used by the dentist). ${ }^{15}$ The PubMed had probing facilities for clinical studies and all publications for all healthcare-related issues, counting drug information. While the Nice and NCCN had EBD guidelines planned by numerous scientific committees and non-useful for primary literature, they are free. It measured the Nice produce medical and dental EBD guidelines, while NCCN offers EBD guidelines about cancer therapy for oral cavity and other oncology conditions and linked management. ${ }^{24,25}$ Some EBD databases comprised a systemic review of dental topics, counting dental medicationrelated issues like Cochrane library, Trip, and Dare. While Trip and Dare more emphasis on cost or PharmacoEconomic for dental medications. ${ }^{34-36}$ Most evidence-based dentistry resources came as a assortment of hundreds or thousands of journals with one searching engine, Ovid, EBSCO, Sage, Springer, Science Direct, Proquest, Willy online library, web of science, and Scopus. ${ }^{42-47,49-51}$ Those databases delimited healthcare information, including dental drug information based on evidence. All of them are paid and want for organizations subscription, not individual.

Two search engines are free of charge and pursuit for all evidence-based dentistry including peer-reviewed primary literature, evidence-based guidelines, EBD textbooks, and masters or doctorate thesis. They are reachable to all dental staff (students, general practitioners, specialists, and consultants); by reviewing previous EBD, the database should be obtainable at healthcare or dental institutions, emphasizing dental college. Five EBD should make accessible all the time. It encompassed PubMed, Nice, NCCN, Google Scholar, and Microsoft Academic. 24,25,53,54 Additionally, the collection of meta-analysis and systemic review studies can be presented by abstract only, free. It should make reachable to all dental specialties. Buying a journal collection depends on several journals, dental specialties, and the USA or European journals. It might get the science director springer, sage, or Willy. Alternatively, it better to choose the web of science and Scopus that has you composed both the USA and European publications. There is one poised might controlled on both sides like EBSCO. All those subscriptions should be organizations based, not individual subscriptions. Some of the EBM resources came as mobile applications (Table 1 and 2). However, other EBM came only as applications like read by QxMD. It is an application probing engine through the collection of journal resemble Ovid, EBSCO, Sage, Springer, Science Direct, Proquest and Willy online library. ${ }^{55}$

\section{CONCLUSION}

The dentists use numerous dental drug resources employed in practice. One of the critical resources was evidence-based dentistry with an emphasis on dental medication information. Some of the resources usually used in the practice which obtainable for all dentists. Others need a subscription. Dental healthcare organizations need implementing the current concept through the local medical library. The dentists should be acquainted with standard evidence-based dentistry drug information references and usage in dental care. As a result, education and training at dental care organizations are recommended to recover the knowledge and practice of dental evidence-based dentistry drug information resources.

\section{ABBREVIATIONS}

KSA: Kingdom of Saudi Arabia; SWOT: Strengths, Weaknesses, Opportunities, and Threats; EBD: Evidence Based Dentistry; NICE: National Institute for health and care excellence; NCCN: National Comprehensive Cancer Network; DARE: Database of Abstracts of Reviews of Effects; ACP: American College of Physicians; BMJ: British Medical Journals.

\section{ACKNOWLEDGEMENT}

None.

\section{CONFLICT OF INTEREST}

The authors declare that there is no conflict of interest.

\section{Funding}

None

\section{Consent for Publications}

Informed consent was obtained from all the participants

\section{Ethical Approval}

This research was exempted from research and ethical committee or an institutional review board (IRB) approval.

https://www.hhs.gov/ohrp/regulations-and-policy/decisioncharts-2018/index.html

\section{ORCID ID}

Yousef Ahmed Alomi (D) https://orcid.org/0000-0003-1381-628X

\section{REFERENCES}

1. Mason J. Medicines optimisation - it's everybody's business. Prescriber. 2013 Apr 5;24(7):40-2. doi: 10.1002/psb.1038.

2. Chauhan N, Moin S, Pandey A, Mittal A, Bajaj U. Indian aspects of drug information resources and impact of drug information centre on community. $J$ Adv Pharm Technol Res. 2013;4(2):84-93. doi: 10.4103/2231-4040.111524, PMID 23833748. Available from: /pmc/articles/ PMC3696227.

3. Alomi YA, Alshammari AM, Saleam Aljohani HS. Dentist's knowledge of evidence-based dentistry and digital applications resources in Saudi Arabia. PTB Reports. 2021;7(1):23-7. doi: 10.5530/PTB.2021.7.5.

4. Frantsve-Hawley J. Evidence locator: sources of evidence-based dentistry information. J Evid Based Dent Pract. 2008 Sep;8(3):133-8. doi: 10.1016/j. jebdp.2008.05.013, PMID 18783754.

5. Palaian S, Mishra P, Shankar PR, Bista D, Purwar B. Contribution of the regional drug information center towards drug safety. JNMA J Nepal Med Assoc. 2006;45(161):216-8. PMID 17160101.

6. Koehler HM. Sources of biomedical information in the dental literature. Drug Inf J. 1975;9(1):30-1. doi: 10.1177/009286157500900105, PMID 10236954

7. Vassilev ZP, Chu AF, Ruck B, Adams EH, Marcus SM. Evaluation of adverse drug reactions reported to a poison control center between 2000 and 2007. Am J Health Syst Pharm. 2009;66(5):481-7. doi: 10.2146/ajhp080267, PMID 19233996.

8. Entezari-Maleki T, Taraz M, Javadi MR, Hajimiri MH, Eslami K, Karimzadeh I, Esmaeili M, Gholami K. A two-year utilization of the pharmacist-operated drug information center in Iran. J Res Pharm Pract. 2014;3(4):117-22. doi: 
10.4103/2279-042X.145368, PMID 25535619.

9. Hands D, Stephens M, Brown D. A systematic review of the clinical and economic impact of drug information services on patient outcome. Pharm World Sci. 2002;24(4):132-8. doi: 10.1023/a:1019573118419, PMID 12227245.

10. Helms MM, Nixon J. Exploring SWOT analysis - where are we now?: a review of academic research from the last decade. Journal of Strategy and Mgt. 2010;3(3):215-51. doi: 10.1108/17554251011064837.

11. Enwere EN, Keating EA, Weber RJ. Balanced scorecards as a tool for developing patient-centered pharmacy services. Hosp Pharm. 2014;49(6):579-84. doi: 10.1310/hpj4906-579, PMID 24958976.

12. Ray S. The risk management process in project management - ProjectManager.com [internet]; 2017. Project Manager [cited Mar 15 2020]. Available from: https://www.projectmanager.com/blog/risk-management-process-steps.

13. Kaplan RS, Mikes A. Managing risks: A new framework [internet]; 2012. Harvard Business Review [cited Mar 15 2020]. Available from: https://hbr.org/2012/06/ managing-risks-a-new-framework.

14. Chiappelli F. Evidence-based dentistry: two decades and beyond. J Evid Based Dent Pract. 2019;19(1):7-16. doi: 10.1016/j.jebdp.2018.05.001, PMID 30926103.

15. Alomi YA, Alshammari AM, Aljohani HSS. Dentist's knowledge of essentia drug information resources in Saudi Arabia. PTB Reports. 2021;7(1):17-22. doi: 10.5530/PTB.2021.7.4.

16. Fineout-Overholt E, Berryman DR, Hofstetter $S$, Sollenberger J. Finding relevant evidence to answer clinical questions. Evid-Based Pract Nurs Healthc. 2011:40-70

17. Sutherland SE. Evidence-based dentistry: Part II. Searching for answers to clinical questions: how to use Medline. J Can Dent Assoc. 2001;67(5):277-80. PMID 11398391

18. Sutherland SE, Walker S. Evidence-based dentistry: Part III. Searching for answers to clinical questions: finding evidence on the Internet. J Can Dent Assoc. 2001;67(6):320-3. PMID 11450294.

19. Malone PM, Kier KL, Stanovich JE. Drug information: A Guide for pharmacists. 3rd ed; 2006.1-877.

20. Gabay M. The clinical practice of drug. Information. 2016.1-539

21. Kier KL, Goldwire M. Drug information resources and literature retrieval. 2018. 619-45.

22. PubMed [internet] [cited Mar 15 2021]. Available from: https://pubmed.ncbi. nIm.nih.gov/.

23. About Medline $®$ and PubMed $®$ : the resources guide [internet] [cited Mar 15 2021]. Available from: https://www.nlm.nih.gov/bsd/pmresources.html.

24. NICE, The National Institute for Health and Care Excellence [internet] [cited Mar 15 2021]. Available from: https://www.nice.org.uk/.

25. NCCN. Evidence-based cancer guidelines, oncology drug compendium, oncology continuing medical education [internet] [cited Mar 15 2021]. Available from: https://www.nccn.org/.

26. About Embase - Biomedical research. Elsevier [internet] [cited Mar 15 2021]. Available from: https://www.elsevier.com/solutions/embase-biomedical-research.

27. Embase coverage and content. Elsevier [internet] [cited Mar 15 2021]. Available from: https://www.elsevier.com/solutions/embase-biomedical-research/ embase-coverage-and-content.

28. ClinicalKey. Lead with answers [internet] [cited Mar 16 2021]. Available from: https://www.clinicalkey.com/\#!

29. Dyna. Med [internet] [cited Jun 21 2021]. Available from: https://www. dynamed.com/.

30. Core EBM. Databases - evidence-based medicine - GSU library research guides at Georgia State University [internet] [cited Mar 15 2021]. Available from: https://research.library.gsu.edu/c.php?g=115558\&p=751758.

31. UpToDate [internet] [cited Mar 15 2021]. Available from: https://www. uptodate. com/contents/search.

32. Cochrane Library [internet] [cited Mar 15 2021]. Available from: https:// www. cochranelibrary.com/cdsr/reviews.

33. Natural Medicines Research Collaboration [internet] [cited Mar 15 2021]. Available from: https://naturalmedicines.therapeuticresearch.com/.

34. Trip Medical Database [internet] [cited Mar 15 2021]. Available from: https:// www.tripdatabase.com/.

35. The database of abstracts of reviews of effects (DARE) [internet] [cited Mar 15 2021]. Available from: https://www.crd.york.ac.uk/crdweb/ShowRecord.asp?!D $=32004000332 \& \mid \mathrm{D}=32004000332$

36. NIHR. Centre for Reviews and Dissemination - CRD Database [internet] [cited Mar 15 2021]. Available from: https://www.crd.york.ac.uk/CRDWeb/.

37. ClinicalTrials.gov [internet] [cited Mar 16 2021]. Available from: https:// clinicaltrials.gov/ct2/home

38. Cochrane handbook for systematic reviews of interventions | cochrane training [internet] [cited Mar 15 2021]. Available from: https://training.cochrane.org/ handbook.

39. Internal medicine books and textbooks. association of clinical pathologists [internet] [cited Mar 15 2021]. Available from: https://www.acponline.org/ clinical-information/journals-publications/books-from-acp.

40. American College of Physicians. association of clinical pathologists [internet]. Internal Medicine [cited Mar 15 2021]. Available from: https://www.acponline. org/.

41. CINAHL complete. EBSCO [internet] [cited Mar 15 2021]. Available from: https://www.ebsco.com/products/research-databases/cinahl-complete.

42. Ovid Database Guide [internet] [cited Mar 15 2021]. Available from: https:// ospguides.ovid.com/OSPguides/embase.htm.

43. EBSCO information services [internet] [cited Mar 15 2021]. Available from: https://www.ebsco.com/

44. EBSCO apps | EBSCO apps and cloud services [internet] [cited Mar 15 2021]. Available from: https://cloud.ebsco.com/apps.

45. About ScienceDirect / Premier platform for discovering peer-reviewed scientific, technical, and medical information. Elsevier [internet] [cited Mar 15 2021]. Available from: https://www.elsevier.com/solutions/sciencedirect.

46. Home - Springer [Internet] [cited Mar 15 2021]. Available from: https://link. springer.com/

47. SAGE Publications Inc | Home [Internet]. [cited 2021 Mar 15]. Available from: https://us.sagepub.com/en-us/nam.

48. ProQuest. Databases, EBooks and Technology for Research [cited Mar 15 2021]. Available from: https://about.proquest.com/.

49. Wiley Online Library | Scientific research articles, journals, books, and reference works [Internet] [cited Mar 15 2021]. Available from: https://onlinelibrary.wiley.com/.

50. Web of Science [internet] [cited Mar 15 2021]. Available from: https://login.webofknowledge.com/error/Error?Error =IPError\&Pathlnfo=\%2F\&RouterURL=h ttps \%3A\%2F\%2Fwww.webofknowl-edge.com\%2F\&Domain=.webofknowledge.com\&Src=IP\&Alias=WOK5

51. Scopus - Abstract and citation database. Elsevier [internet] [cited Mar 15 2021]. Available from: https://www.elsevier.com/solutions/scopus.

52. The BMJ | The BMJ: leading general medical journal. Research. Educ Comment.

53. Google Scholar [internet] [cited Mar 16 2021]. Available from: https://scholar. google.com/schhp?hl=en

54. Home | Microsoft Academic [Internet] [cited Mar 16 2021]. Available from: https://academic.microsoft.com/home.

55. Read by QxMD [internet] [cited Jun 22 2021]. Available from: https://apps. apple.com/sa/app/read-by-qxmd/id574041839?!=ar.

56. Clinical resources and products | adult immunization. association of clinical pathologists [internet] [cited Mar 15 2021]. Available from: https://www.acponline. org/clinical-information/clinical-resources-products.

57. Google Scholar citations help [internet] [cited Mar 16 2021]. Available from: https://scholar.google.com/int//en/scholar/citations.html. 